\title{
On the CR-Structure of Certain Linear Group Orbits in Infinite Dimensions
}

\author{
WILHELM KAUP
}

\begin{abstract}
For large classes of complex Banach spaces (mainly operator spaces) we consider orbits of finite rank elements under the group of linear isometries. These are (in general) real-analytic submanifolds of infinite dimension but of finite CR-codimension. We compute the polynomial convex hull of such orbits $M$ explicitly and show as main result that every continuous CR-function on $M$ has a unique extension to the polynomial convex hull which is holomorphic in a certain sense. This generalizes to infinite dimensions results from a recent joint paper with D. Zaitsev in Inventiones math. 153, 45-104.
\end{abstract}

Mathematics Subject Classification (2000): 32V25 (primary); 17C50, 32H02, 32E20, 32M15, 46G20 (secondary).

The purpose of this note is to partially extend the results of [13] to infinite dimensions. We illustrate this for the simplest possible example: let $E$ be a complex Hilbert space of infinite or of finite dimension $\geq 2$ with open unit ball $B$ and unit sphere $S$. Let $K$ be the group of all surjective linear isometries of $E$ (the unitary group of $E$ ). Then $S$ is a $K$-orbit in $E$. From the classical maximum principle it is obvious that if $D \subset \mathbb{C}$ is any domain, then every holomorphic map $D \rightarrow E$ with image in $S$ is constant. On the other hand, there are many holomorphic functions on $S$ in the sense that they satisfy the tangential Cauchy-Riemann differential equations (instead of holomorphic these are usually called $C R$-functions): $S$ is obviously a real-analytic submanifold of $E$ with (real) tangent space $T_{a} S=\{z \in E: \Re(z \mid a)=0\}$ at every $a \in S$. The maximal complex linear subspace of $E$ contained in $T_{a} S$ clearly is $H_{a} S:=$ $T_{a} S \cap i T_{a} S=\{z \in E:(z \mid a)=0\}$, called the holomorphic tangent space to $S$ at $a$. Now for every complex Banach space $F$ the smooth function $f: S \rightarrow F$ is called $C R$ if for every $a \in S$ the differential $d f_{a}: T_{a} S \rightarrow F$ is complex linear on the subspace $H_{a} S \subset T_{a} S$. A continuous function $S \rightarrow F$ is called $C R$ if it

Partially supported by D.G.I. project no. BFM2002-01529, Spain.

Pervenuto alla Redazione il 13 ottobre 2003 e in forma definitiva il 5 aprile 2004. 
is locally the uniform limit of a sequence of smooth CR-functions, a concept, that in finite dimensions (on arbitrary CR-manifolds) is equivalent to the more traditional requirement, that CR-functions satisfy the tangential Cauchy-Riemann differential equations in the distribution sense, see [2] for this and [3], [1] as general references for CR-manifolds and CR-functions.

In case $\operatorname{dim}(E)<\infty$ it is well known that the continuous $\mathbb{C}$-valued CRfunctions on the unit sphere $S \subset E$ are precisely those functions $f$ on $S$ that have a continuous extension to the closed unit ball $\bar{B}$ with $\left.f\right|_{B}$ holomorphic. In case $E$ has infinite dimension it is not too difficult to get the same statement by a reduction argument to the finite dimensional case. In the present paper we get similar results for a large class of infinite dimensional CR-manifolds that occur in certain complex Banach spaces $E$ as orbits of the connected identity component $K$ of the full group of isometries of $E$. These $E$ all have the property that their open unit ball is homogeneous under its biholomorphic automorphism group and hence is a bounded symmetric domain. These Banach spaces come with a canonical Jordan algebraic structure that is heavily exploited.

For instance, the following examples occur in our treatment: let $H, L \neq 0$ be complex Hilbert spaces with $H$ of infinite dimension and let $E:=\mathcal{L}(L, H)$ be the complex Banach space of all bounded linear operators $L \rightarrow H$ (which is a Hilbert space only in case $\operatorname{dim}(L)=1)$. Denote by $K$ the connected identity component of the group of all linear isometries of $E$. Then $K$ consists of all transformations $z \mapsto u z v$, where $u, v$ are unitary operators on $L, H$ respectively. Fix an operator $a \in E$ of finite rank $r \geq 1$ and denote by $M:=K(a)$ the corresponding $K$-orbit. Then $M \subset E$ is a real-analytic CR-submanifold of infinite dimension and finite CR-codimension $\geq r$. We compute the polynomial convex hull $\mathcal{Z}$ of $M$ in $E$ explicitly, and our main result (Theorem 7.5) states in this special case that every continuous CR-function with values in an arbitrary complex Banach space $F$ has a unique continuous extension to $\mathcal{Z}$ which in a certain sense is holomorphic on the interior of $\mathcal{Z}$.

\section{1. - Preliminaries}

For Banach spaces $E, F$ over the field $\mathbb{K}=\mathbb{C}$ (or $\mathbb{K}=\mathbb{R}$ ) we denote by $\mathcal{L}(E, F)$ the $\mathbb{K}$-Banach space of all bounded (= continuous) linear operators $E \rightarrow F$ and by $\mathcal{K}(E, F)$ the linear subspace of all compact operators. In case $E=F$ we also write $\mathcal{L}(E)$ as well as $\mathcal{K}(E)$ instead of $\mathcal{L}(E, E)$ and $\mathcal{K}(E, E)$. The group of all invertible operators in the Banach algebra $\mathcal{L}(E)$ is denoted by $\mathrm{GL}(E)$. By $E^{*}:=\mathcal{L}(E, \mathbb{K})$ we denote the dual of $E$. We always consider $E$ in the canonical way as closed linear subspace of the bidual $E^{* *}$.

For a Banach space $E$ we call a smooth Banach manifold $M$ together with an injective smooth mapping $\varphi: M \rightarrow E$ an immersed submanifold of $E$ if for every $a \in M$ the corresponding differential $d \varphi_{a}: T_{a} M \rightarrow T_{\varphi(a)} E \approx$ $E$ is injective. In this sense every subset $S \subset E$ occurs as (0-dimensional) 
immersed submanifold of $E$ with respect to the discrete topology. We avoid trivial situations like this by considering mainly connected manifolds $M$.

For every immersed submanifold $M$ of the complex Banach space $E$ and every $a \in M$ the tangent space $T_{a} M$ at $a$ to $M$ is a real (complete normable) topological vector space that we may consider as an $\mathbb{R}$-linear subspace of $E$ (not necessarily having the induced topology). In this sense, $H_{a} M:=T_{a} M \cap i T_{a} M$, called the holomorphic tangent space to $M$ at the point $a$, is the biggest $\mathbb{C}$-linear subspace contained in $T_{a} M$. In a similar way we let $\mathbb{C} T_{a} M:=\left(T_{a} M \oplus i T_{a} M\right) / L$ for $L=\left\{(x, i y): x, y \in H_{a} M\right.$ with $\left.x+i y=0\right\}$ be the 'smallest complex linear space' containing $T_{a} M$ as closed $\mathbb{R}$-linear subspace.

The immersed submanifold $\varphi: M \rightarrow E$ of $E$ is called direct if for every $a \in M$ the tangent space $T_{a} M$ is closed in $E$ and admits a closed $\mathbb{R}$-linear complement in $E$. If in addition $\varphi$ is a homeomorphism onto its image we identify $M$ with $\varphi(M)$ and call it a direct submanifold of $E$.

For complex Banach spaces $E, F$ a smooth function $f: U \rightarrow F, U \subset E$ open, is called holomorphic if the (R-linear) Fréchet derivative $d f_{a}: E \rightarrow F$ actually is $\mathbb{C}$-linear for every $a \in U$, see [5] or [7] as reference for infinite dimensional holomorphy. A complex Banach manifold is a manifold locally modeled over open subsets of complex Banach spaces with biholomorphic transition functions. For complex Banach manifolds $X, Y$ and every subset $S \subset X$ we call a mapping $f: S \rightarrow Y$ pull-back holomorphic if $f$ is continuous and if for every complex Banach manifold $Z$ and every holomorphic mapping $\varphi: Z \rightarrow X$ with $\varphi(Z) \subset S$ the pull-back $f \circ \varphi: Z \rightarrow Y$ also is holomorphic. A subset $C \subset S$ is called a holomorphic arc component of $S \subset X$ if the following two conditions are satisfied, where $\Delta \subset \mathbb{C}$ is the open unit disk:

(i) $C \neq \emptyset$, and $\varphi(\Delta) \subset C$ for every holomorphic mapping $\varphi: \Delta \rightarrow X$ with $\varphi(\Delta) \subset S$ and $\varphi(\Delta) \cap C \neq \emptyset$,

(ii) $C$ is minimal with respect to property (i).

Clearly, $S$ is the disjoint union of its holomorphic arc components, and for every connected complex Banach manifold $Z$ every holomorphic map $\varphi: Z \rightarrow X$ with $\varphi(Z) \subset S$ has its image in a holomorphic arc component of $S$. Finally, for every subset $S$ of a complex Banach space $E$ by

$$
\begin{aligned}
\operatorname{pch}(S):= & \{z \in E:|f(z)| \leq \sup |f(S)| \text { for all holomorphic polynomials } \\
& f: E \rightarrow \mathbb{C}\} \\
\operatorname{hch}(S):= & \{z \in E:|f(z)| \leq \sup |f(S)| \text { for all holomorphic functions } f: E \rightarrow \mathbb{C}\}
\end{aligned}
$$

we denote the polynomial convex hull and the holomorphic convex hull of $S$, where we allow $\sup |f(S)|=+\infty$ if $f(S) \subset \mathbb{C}$ is unbounded. Clearly, $\operatorname{pch}(S)$ contains $\mathrm{hch}(S)$ and is contained in the closed (linear) convex hull $\operatorname{cch}(S)$ of $S$. Due to the power series expansion of holomorphic functions on $E$ polynomial and holomorphic convex hulls of relatively compact subsets $S \subset E$ coincide. 


\section{2. - JB*-triples}

In the following we recall some basic facts on $\mathrm{JB}^{*}$-triples. These are the complex Banach spaces in which we want to study certain orbits under its group of isometries. We start with a slightly more general structure.

Definition 2.1. A complex Banach space $E$ together with a continuous map

$$
E \times E \times E \rightarrow E, \quad(x, y, z) \mapsto\{x y z\}
$$

is called a $\mathrm{J}^{*}$-triple (compare [9]), if for all $a, b, x, y, z \in E$ the following conditions hold

(i) $\{x y z\}$ is symmetric bilinear in the outer variables $(x, z)$ and conjugate linear in the inner variable $y$.

(ii) $\{a b\{x y z\}\}=\{\{a b x\} y z\}-\{x\{b a y\} z\}+\{x y\{a b z\}\}$.

(iii) The operator $L_{a} \in \mathcal{L}(E)$ defined by $c \mapsto\{a a c\}$ is hermitian, i.e. $\exp \left(i t L_{a}\right) \in$ $\mathrm{GL}(E)$ is an isometry for every $t \in \mathbb{R}$.

More generally, we denote for every $a, b \in E$ by $L(a, b) \in \mathcal{L}(E)$ the operator $z \mapsto\{a b z\}$ and by $Q_{a}$ the antilinear endomorphism $z \mapsto\{a z a\}$, that is, $L_{a}=L(a, a)$. The (not necessarily closed) linear subspace $I \subset E$ is called a subtriple if $\{I I I\} \subset I$ holds, and $I$ is called an ideal in $E$ if even $\{E E I\}+\{E I E\} \subset I$ is true. The $\mathrm{J}^{*}$-triple is called irreducible if it is not the direct sum of two nonzero closed ideals.

The $J^{*}$-triples form a category in an obvious way - the morphisms are the bounded linear operators $g$ satisfying

$$
g\{x y z\}=\{(g x)(g y)(g z)\}
$$

for all $x, y, z$ in the domain of $g$. We always denote by

$$
\operatorname{Aut}(E):=\{g \in \mathrm{GL}(E): g \text { morphism }\}
$$

the automorphism group of $E$, which is a real algebraic subgroup of $\mathrm{GL}(E)$ in the sense of [8]. In particular, Aut $(E)$ is a real Banach Lie group in the norm topology of $\mathcal{L}(E)$. The corresponding Lie algebra is the derivation algebra

$$
\begin{aligned}
& \operatorname{Der}(E):=\{\delta \in \mathcal{L}(E): \delta\{x y z\}=\{(\delta x) y z\}+\{x(\delta y) z\}+\{x y(\delta z)\} \\
&\text { for all } x, y, z \in E\} .
\end{aligned}
$$

Besides the automorphism group also the structure group $\operatorname{Str}(E)$ of $E$ is of importance, which is defined as follows, compare [14]: let $\bar{E}$ be the J*-triple obtained from $E$ by changing the complex structure to its conjugate one and keeping the triple product. Then

$$
\begin{aligned}
\operatorname{Str}(E):=\{(g, h) \in \mathrm{GL}(E) \times \mathrm{GL}(\bar{E}) & : g\{x y z\}=\{(g x)(h y)(g z)\} \\
& \text { and } h\{x y z\}=\{(h x)(g y)(h z)\} \text { for all } x, y, z\}
\end{aligned}
$$


is a complex Banach Lie subgroup of $\mathrm{GL}(E) \times \mathrm{GL}(\bar{E})$ with respect to the norm topology.

Definition 2.2. A $\mathrm{J}^{*}$-triple $E$ is called a $\mathrm{JB}^{*}$-triple if for all $a \in E$

(i) the hermitian operator $L_{a}$ has real spectrum $\geq 0$ and

(ii) $\|\{a a a\}\|=\|a\|^{3}$.

A complex Banach space $E$ carries the structure of a $\mathrm{JB}^{*}$-triple if and only if the open unit ball $B$ of $E$ is homogeneous under its $\operatorname{group} \operatorname{Aut}(B)$ of all biholomorphic transformations, and then the triple product is uniquely determined by the norm of $E$. In the following, $E$ will always be a JB*triple. The real Banach Lie group $\operatorname{Aut}(E)$ of all automorphisms is a real Banach Lie group that coincides with the group of all linear surjective isometries of $E$. With $K:=\operatorname{Aut}(E)^{0}$ we denote its connected identity component. The canonical projection $\mathrm{GL}(E) \times \mathrm{GL}(\bar{E}) \rightarrow \mathrm{GL}(E)$ induces a biholomorphic group isomorphism from the structure group $\operatorname{Str}(E)$ onto a closed complex Banach Lie subgroup of $\mathrm{GL}(E)$. We may therefore consider $\operatorname{Str}(E)$ as a complex Lie subgroup of $\mathrm{GL}(E)$ and denote by $\Gamma:=\operatorname{Str}(E)^{0}$ its connected identity component in the following. The Lie algebra of $\Gamma$ is $\operatorname{Der}(E) \oplus i \operatorname{Der}(E)$ and $K \subset \Gamma$ is a real form of $\Gamma$.

We are interested in various structures on the orbits $M:=K(a)$ and $X:=$ $\Gamma(a), a \in E$. Clearly, the isotropy subgroup $K_{a}:=\{g \in K: g(a)=a\}$ is closed in $K$ and the orbital map $K / K_{a} \rightarrow M$ given by $g \mapsto g(a)$ is a continuous bijection. In case $K_{a}$ is a direct real-analytic subgroup of $K$, as a consequence of Godement's theorem, the quotient $K / K_{a}$ has the structure of a real-analytic (Banach) manifold in such a way that the canonical projection $K \rightarrow K / K_{a}$ is a real-analytic submersion. Furthermore, $K / K_{a}$ then is an immersed realanalytic submanifold of $E$ via the orbital map. The same holds for the complex (Banach) manifold $\Gamma / \Gamma_{a}$ if the isotropy subgroup $\Gamma_{a}$ is direct in $\Gamma$.

\section{3. - (Von Neumann) regular orbits}

Fix a $\mathrm{JB}^{*}$-triple $E$ in the following. The elements $a, b \in E$ are called (triple) orthogonal, and we write $a \perp b$, if $L(a, b)=0$ is satisfied. Then $a \perp b$ and $b \perp a$ are equivalent conditions. Every odd continuous function $f: \mathbb{R} \rightarrow \mathbb{R}$ induces an odd continuous function $f: E \rightarrow E$ (odd functional calculus, compare [11]) with $\boldsymbol{f}(a+b)=\boldsymbol{f}(a)+\boldsymbol{f}(b)$ if $a \perp b$. For instance, if $f \in \mathbb{R}[t]$ is an odd polynomial, $f(a)=p\left(L_{a}\right)(a)$, where $p \in \mathbb{R}[t]$ satisfies $f(t)=t p\left(t^{2}\right)$. In particular, $f(a)=\{a a a\}$ for the cube function $f(t)=t^{3}$. The fixed points in $E$ of the cube function, i.e. the elements $e \in E$ with $\{e e e\}=e$, are called tripotents. For tripotents $e, c \in E$ we write $e \leq c$ if $c-e$ is a tripotent orthogonal to $e$. The identity $f(t e)=f(t) e$ holds for all $t \in \mathbb{R}$ 
and all tripotents $e \in E$. Every tripotent $e \in E$ induces a Peirce decomposition

$$
E=E_{1}(e) \oplus E_{1 / 2}(e) \oplus E_{0}(e),
$$

where $E_{k}(e)$ is the $k$-eigenspace in $E$ of the operator $L_{e}$ for every $k \in \mathbb{R}$. Furthermore,

$$
A(e):=\{z \in E:\{e z e\}=z\}
$$

is a JB-algebra with unit $e$ with respect to product $x \circ y:=\{x e y\}$, satisfying

$$
E_{1}(e)=A(e) \oplus i A(e) .
$$

Every tripotent $e \neq 0$ with $A(e)=\mathbb{R} e$ is called minimal.

Definition 3.2. For every $a \in E$ denote by $[a]$ the smallest closed subtriple of $E$ containing $a$ and call $a \in E$ (von Neumann) regular, compare [14, p. 42] and [11, Section 4], if one of the following equivalent conditions is satisfied:

(i) $\{a b a\}=a$ for some $b \in E$,

(ii) the restriction of $L_{a}$ to $[a]$ is an invertible operator on $[a]$,

(iii) there is a tripotent $e \in[a]$ with $a \in A(e)$.

For the element $a \in E$ the element $b$ in (i) can be chosen uniquely from $[a]$ and the tripotent $e$ in (iii) can be chosen uniquely in such a way that $a$ is a square in the JB-algebra $A(e)$. Indeed, for every $a \in E$ there exists a unique compact subset $\Omega \subset \mathbb{R}$ with $0 \in \Omega=-\Omega$ together with a unique triple isomorphism $\varphi$ from $[a]$ onto the $\mathrm{JB}^{*}$-triple $\mathcal{C}^{-}(\Omega)$ of all odd continuous functions $\Omega \rightarrow \mathbb{C}$ such that $\varphi(a)=\mathrm{id}_{\Omega}$. Then $a$ is regular if and only if 0 is isolated in $\Omega$.

PROPOSITION 3.3. For every regular $a \in E$ the operator $L_{a}$ has closed image $\operatorname{im}\left(L_{a}\right)$ in $E$ and

$$
E=\operatorname{im}\left(L_{a}\right) \oplus \operatorname{ker}\left(L_{a}\right)
$$

holds as direct sum of closed linear subspaces. Furthermore, the orbit $X:=\Gamma(a)$, $\Gamma=\operatorname{Str}(E)^{0} \subset \mathrm{GL}(E)$, is a locally closed direct complex submanifold of $E$ with tangent space $T_{a} X=\operatorname{im}\left(L_{a}\right)$ at $a \in X$. Every $x \in X$ is regular and $\Gamma$ acts transitively as holomorphic transformation group on $X$.

Proof. Fix a tripotent $e \in[a]$ with $a \in A(e)$. Then

$$
\operatorname{im}\left(L_{a}\right)=E_{1}(e) \oplus E_{1 / 2}(e), \quad \operatorname{ker}\left(L_{a}\right)=E_{0}(e)
$$

together with (3.1) shows the first claim. For shorter notation put $T:=\operatorname{im}\left(L_{a}\right)$, $N:=\operatorname{ker}\left(L_{a}\right)$ for a while and define a holomorphic map $\varphi: E \rightarrow E$ by $\varphi(t+n):=h_{t}(a+n)$ for all $t \in T$ and all $n \in N$, where $h_{t}:=\exp (L(t, a))$. Because of $h_{t} \in \Gamma$ for all $t$ we have $\varphi(T) \subset X$. Now $\varphi$ has invertible derivative at $0 \in E$, by the implicit function theorem we may therefore assume that the restriction of $\varphi$ to $U \oplus V$ is biholomorphic to an open neighbourhood of $a=\varphi(0)$, where $U, V$ are suitable open neighbourhoods of the origins in $T$ and $N$ respectively. The restriction of $L_{a}$ to $[a]$ has spectrum $>0$. Therefore, the element $a$ is isolated in $\varphi(N)=a+N$, and we may assume without loss of generality that $\varphi(V) \cap X=\{a\}$, that is, $\varphi(U \oplus V) \cap X=\varphi(U)$ and $X$ is a direct complex submanifold of $E$ with tangent space $T$ at $a$. 


\section{4. - Algebraic orbits}

The element $a \in E$ is called algebraic, if $p(a)=0$ holds for some nonzero odd polynomial $p \in \mathbb{R}[t]$, or equivalently, if the closed complex subtriple $[a] \subset$ $E$ generated by $a$ has finite dimension. This is also equivalent to the existence of a representation

$$
a=\lambda_{1} e_{1}+\lambda_{2} e_{2}+\ldots+\lambda_{s} e_{s}
$$

with pairwise orthogonal tripotents $e_{j} \neq 0$ and real coefficients satisfying

$$
\|a\|=\lambda_{1}>\lambda_{2}>\ldots>\lambda_{s}>0 .
$$

Clearly, every algebraic element is regular. The representation (4.1) is uniquely determined by the algebraic element $a$. For instance, the squares of the coefficients $\lambda_{k}(a):=\lambda_{k}$ are precisely the eigenvalues of the operator $L_{a}$ restricted to $[a]$. Also, every tripotent $e_{k}$ can be recovered from $a$ in the form $e_{k}=f(a)$, where $f: \mathbb{R} \rightarrow \mathbb{R}$ is an arbitrary odd continuous function satisfying $f\left(\lambda_{j}\right)=\delta_{j k}$ for $j=1, \ldots, s$.

For the rest of the section let $a \in E$ satisfy (4.1) and set $\lambda_{0}:=0$ as well as $\lambda_{-j}:=-\lambda_{j}$ for $j=1, \ldots, s$. Following [13] we define operators on $E$ by

$$
\Phi_{a}:=2\left(L_{a}+Q_{a}\right), \quad \Psi_{a}:=2\left(L_{a}-Q_{a}\right) \text { and } \Theta_{a}:=\Phi_{a} \Psi_{a}=4\left(L_{a}^{2}-Q_{a}^{2}\right) .
$$

Also we put

$$
N_{a}:=\operatorname{ker}\left(\Psi_{a}\right), \quad T_{a}:=\operatorname{im}\left(\Psi_{a}\right) \text { and } H_{a}:=\operatorname{im}\left(\Theta_{a}\right) .
$$

Clearly $a \in N_{a}, i a \in T_{a}$ and $a \notin H_{a}$. We will see below that $T_{a}$ is the tangent space and $N_{a}$ will be sort of a normal space to the $K$-orbit through $a$.

For all $j, k \in \mathbb{Z}$ with $|j| \leq k \leq s$ consider the refined Peirce space

$$
E^{j k}=E^{j k}(a):=\left\{z \in E: 2 L_{a} z=\left(\lambda_{j}^{2}+\lambda_{k}^{2}\right) z \text { and } Q_{a} z=\lambda_{j} \lambda_{k} z\right\} .
$$

Then we have the refined Peirce decompositions

$$
E=\bigoplus_{|j| \leq k \leq s} E^{j k}, \quad N_{a}=\bigoplus_{j=0}^{s} E^{j j} \text { and } T_{a}=\bigoplus_{j<k} E^{j k},
$$

and the following statement is obvious.

Lemma 4.5. $\quad E=T_{a} \oplus N_{a}$ is a direct sum of closed $\mathbb{R}$-linear subspaces. Furthermore, $H_{a}$ is the maximal complex linear subspace of $T_{a}$. The restrictions of the $\mathbb{R}$-linear operator $\Psi_{a}$ and of the $\mathbb{C}$-linear operator $\Theta_{a}$ to their respective images are invertible.

For use in the following proposition we state for $N_{a}=N_{a}+a$ 
LEMMA 4.6. The intersection of the orbit $M=K(a)$ with $N_{a}$ contains a as isolated point.

ProOF. Fix an $\varepsilon>0$ with $\varepsilon<\left(\lambda_{k}-\lambda_{j}\right)$ for all $k>j \geq 0$. Assume that $c \in\left(M \cap N_{a}\right)$ satisfies $\|a-c\|<\varepsilon$. Fix $j \geq 0$ and denote by $a_{j}, c_{j} \in E^{j j}$ the corresponding components of $a, c$ with respect to the direct sum decomposition of $N_{a}$ in (4.4). Clearly $a_{j}=\lambda_{j} e_{j}$ if we put $e_{0}:=0 \in E$. Since $c$ is in $M$, the element $c_{j}$ is a linear combination of orthogonal tripotents in $E^{j j}$ with certain coefficients from $\left\{\lambda_{1}, \ldots, \lambda_{s}\right\}$. Denote by $F \subset E^{j j}$ the closed real subtriple generated by $a_{j}$ and $c_{j}$. Then $F$ can be identified with the real Banach algebra $\mathcal{C}_{0}(\Omega, \mathbb{R})$ of all continuous real functions vanishing at infinity on a suitable locally compact topological space $\Omega$ in such a way that $a_{j} \equiv \lambda_{j}$. But the function $a_{j}-c_{j}$ takes only values from $\left\{\lambda_{j}-\lambda_{k}:-s \leq k \leq s\right\}$, which implies $a_{j}=c_{j}$ because of $\left\|a_{j}-c_{j}\right\|<\varepsilon$. This implies $a=c$ since $j$ was arbitrarily chosen.

PROPOSITION 4.7. Let $f: \mathbb{R} \rightarrow \mathbb{R}$ be an odd continuous function having only isolated zeros in $\mathbb{R}$. Then

$$
S:=\{a \in E: f(a)=0\}
$$

is a closed direct real-analytic submanifold of $E$. Every connected component $M$ of $S$ is an orbit of the linear group $K:=\operatorname{Aut}(E)^{0}$. Every $a \in S$ is algebraic and $T_{a}$ as defined in (4.3) is the tangent space to $S$ at $a$.

Proof. Fix $a \in S$. Since $f$ has only finitely many zeros in the interval $[0,\|a\|]$ there exists a representation (4.1) for $a$ and hence $a$ is algebraic. Define a real-analytic map $\varphi: E \rightarrow E$ by $\varphi(t+n):=k_{t}(a+n)$ for all $t \in T_{a}$ and all $n \in N_{a}$, where $k_{t}:=\exp (L(t, a)-L(a, t))$. Then $k_{t} \in K$ for all $t$ and with Lemma 4.6 it is shown as in Proposition 3.3 that $S$ is a real-analytic submanifold of $E$ around $a$ with tangent space $T_{a} S=T_{a}$. Since the orbit $K(a)$ is a neighbourhood of $a$ in $S$ and $a \in S$ was arbitrarily chosen, the connected group $K$ acts transitively on every connected component of $S$.

Proposition 4.7 for the special function $f(t)=t^{3}-t$ (i.e. $S$ is the subset of all tripotents in $E$ ) is already contained in [16, Satz 4.4].

It is easily seen that for the algebraic element $a$ with representation (4.1) and tripotent $e:=e_{1}+\ldots+e_{s}$ we have

$$
E^{00}=E_{0}(e) \text { and } E^{j j}=A\left(e_{j}\right) \text { if } j>0 .
$$

In particular

$$
A(a):=\bigoplus_{j=1}^{s} A\left(e_{j}\right)
$$

is a JB-subalgebra of $A(e)$ with unit $e$. For the complex linear operator $P_{a}:=$ $Q_{e} Q_{a}$ put

$$
E_{1}(a):=\operatorname{ker}\left(L_{a}-P_{a}\right), \quad E_{1 / 2}(a):=\operatorname{im}\left(L_{a}-P_{a}\right) \text { and } E_{0}(a):=E_{0}(e) .
$$


Then we have the generalized Peirce decomposition for the algebraic element $a \in E$

$$
E=E_{1}(a) \oplus E_{1 / 2}(a) \oplus E_{0}(a)
$$

with

$$
\begin{aligned}
E_{1}(a) & =A(a) \oplus i A(a), \quad N_{a}=A(a) \oplus E_{0}(a) \text { and } \\
T_{a} & =i A(a) \oplus E_{1 / 2}(a)=\bigoplus_{j=1}^{s}\left(A\left(e_{j}\right) \oplus E_{1 / 2}\left(e_{j}\right)\right) .
\end{aligned}
$$

Consider again an algebraic element $a \in E$ with representation (4.1). Then the $s$-fold $\ell^{\infty}$-sum $E^{s}$ is also a $\mathrm{JB}^{*}$-triple and we have the diagonal action of $K=\operatorname{Aut}(E)^{0}$ on it, i.e. $g\left(z_{1}, \ldots, z_{s}\right)=\left(g z_{1}, \ldots, g z_{s}\right)$. It is easily seen that

$$
S:=\left\{\left(c_{1}, \ldots, c_{s}\right) \in E^{s}: c_{j} \text { pairwise orthognonal tripotents in } E\right\}
$$

is a real-analytic submanifold of $E^{s}$ and that every connected component of $S$ is a $K$-orbit. Let $\tilde{e}:=\left(e_{1}, \ldots, e_{s}\right)$ and put $\tilde{M}:=K(\tilde{e})$. Then the tangent space to $\tilde{M}$ at $\tilde{e}$ can easily be computed. The mapping $\varphi: \tilde{M} \rightarrow M$ defined by $\varphi\left(c_{1}, \ldots, c_{s}\right)=\lambda_{1} c_{1}+\ldots+\lambda_{s} c_{s}$ is a real-analytic diffeomorphism. $\varphi$ is CR, but its inverse in general is not CR.

Every orbit $M=K(a), a \in M$, has the structure of a homogeneous $K$ space. For every odd continuous function $f: \mathbb{R} \rightarrow \mathbb{R}$ and $b:=\boldsymbol{f}(a)$ then $\boldsymbol{f}$ induces a continuous $K$-equivariant mapping from $M$ onto the orbit $N:=K(b)$. In case $a$ is algebraic also $b$ has the same property and $f: M \rightarrow N$ is a real-analytic fiber bundle, compare [13] for the finite dimensional case. The complex subbundle $H M \subset T M$ gives a contact structure on $M$. As in [13, (7.9)] it is seen that $f: M \rightarrow N$ always is a contact transformation (i.e. its differential maps $H M$ into $H N$ ). On the other hand, in case $E$ is irreducible, $f: M \rightarrow N$ is a CR-mapping (i.e. a contact transformation whose differential is complex linear on every holomorphic tangent space $\left.H_{x} M, x \in M\right)$ if and only if $f\left(\lambda_{j}(a)\right)=c \lambda_{j}(a)$ for all $j$ and some real constant $c \geq 0$, i.e. the orbits differ by the homothety with the factor $c$. As an extension of [13] we therefore ask: when are the orbits $K(a), K(b) C R$-equivalent in case $a, b \in E$ are algebraic?

\section{5. - Some counterexamples}

What happens if in Proposition 4.7 the continuous odd function $f$ is allowed to have accumulation points of its zeros? We show that then $S$ in general no longer is a submanifold of $E$ in the induced topology.

For this let $H$ be a complex Hilbert space of infinite dimension with inner product $(z \mid w)$ that is complex linear in the first variable and put $E:=\mathcal{L}(H)$. 
For every pair $x, y$ of unit vectors in $H$ let $x \otimes y^{*} \in E$ be defined as the rank one operator $h \mapsto(h \mid y) x$. Then $x \otimes y^{*}$ is a minimal tripotent in $E$ and every minimal tripotent in $E$ occurs this way. The group $K=\operatorname{Aut}(E)^{0}$ consists of all transformations $z \mapsto u z v^{*}$ with $u, v$ unitary operators on $H$.

Now fix a family $\left(e_{\lambda}\right)_{\lambda \in \Lambda}$ of pairwise orthogonal minimal tripotents in $E$, where $\Lambda \subset \mathbb{R}$ is an infinite subset with $0<\Lambda \leq 1$. Then the infinite sum

$$
a:=\sum_{\lambda \in \Lambda} \lambda e_{\lambda}
$$

converges in the $w^{*}$-topology to an element $a \in E$ with $\|a\| \leq 1$. Assume that there is an orthonormal basis $\left(x_{\lambda}\right)_{\lambda \in \Lambda}$ of $H$ with $e_{\lambda}=x_{\lambda} \otimes x_{\lambda}^{*}$ for all $\lambda \in \Lambda$. Then the isotropy subgroup $K_{a}$ consists of all transformations $z \mapsto u z u^{*}$, where $u$ runs over all unitary operators on $H$ that are diagonal with respect to the basis $\left(x_{\lambda}\right)$ (i.e. have every $x_{\lambda}$ as eigen vector). It is easily seen that $K_{a}$ is a direct subgroup of $K$, i.e. $M$ is an immersed real-analytic submanifold of $E$ (with respect to the orbital map $K / K_{a} \rightarrow M$ ). The tangent space at $a$ is given by

$$
T_{a} M=\{i(u a-a v): u, v \in \mathcal{H}\},
$$

where $\mathcal{H} \subset E=\mathcal{L}(H)$ is the $\mathbb{R}$-linear subspace of all hermitian operators. Consider the direct sum decomposition into $w^{*}$-closed $\mathbb{R}$-linear subspaces

$$
E=N_{a} \oplus R_{a},
$$

where

$$
N_{a}:=\operatorname{ker}\left(L_{a}-Q_{a}\right)=\left\{z \in E:\left(z e_{\lambda} \mid e_{\mu}\right) \in \delta_{\lambda \mu} \mathbb{R} \text { for all } \lambda, \mu \in \Lambda\right\}
$$

and

$$
R_{a}:=\left\{z \in E:\left(z e_{\lambda} \mid e_{\lambda}\right) \in i \mathbb{R} \text { for all } \lambda \in \Lambda\right\} .
$$

The inclusion $T_{a} M \subset R_{a}$ is easily seen, but equality does not hold in general. This happens for instance, if $a$ is not regular, that is, if inf $\Lambda=0$. In any case, for every $\varepsilon>0$ there exists an element $a_{\varepsilon} \in N_{a} \cap M$ with $0<\left\|a-a_{\varepsilon}\right\|<\varepsilon$. Indeed, choose $\lambda, \mu \in \Lambda$ with $0<(\lambda-\mu)<\varepsilon$ and put $a_{\varepsilon}:=a+(\lambda-\mu)\left(e_{\mu}-e_{\lambda}\right)$. As a consequence, the induced topology from $E$ to $M$ is not locally connected and, in particular, the orbital map $K / K_{a} \rightarrow M$ is not a homeomorphism onto its image, and $M$ is not a submanifold of $E$.

As a second example consider the (commutative) $\mathrm{C}^{*}$-algebra $E=\mathcal{C}(\Omega)$ of all continuous complex valued functions on a compact connected topological space $\Omega$ that contains more than one point. Then $E$ also is a $\mathrm{JB}^{*}$-triple with respect to $\{f g h\}=f \bar{g} h$. Fix a function $a \not \equiv 0$ in $E$. Then $a$ is regular in $E$ if and only if $a(\omega) \neq 0$ for all $\omega \in \Omega$. The group $\Gamma$ consists of all transformations $z \mapsto e^{g} z$ with $g \in E$. We know from Proposition 3.3 that the orbit $X:=\Gamma(a)$ is a locally closed direct complex submanifold of $E$ if $a$ is regular. Otherwise, there exists $\omega_{0} \in \Omega$ with $z\left(\omega_{0}\right)=0$ for all $z \in X$ and hence $X$ not even is locally closed in $E$. Also, in this case $\operatorname{im}\left(L_{a}\right)$ is not closed in $E$ and $\operatorname{im}\left(L_{a}\right)+\operatorname{ker}\left(L_{a}\right)$ is a proper subspace of $E$. 


\section{6. - Finite rank orbits}

For the rest of this section let us assume that $a \in E$ has finite rank $r$, that is, $a$ has a representation

$$
a=\sigma_{1} e_{1}+\sigma_{2} e_{2}+\ldots+\sigma_{r} e_{r}
$$

with uniquely determined real coefficients

$$
\sigma_{1} \geq \sigma_{2} \geq \ldots \geq \sigma_{r}>0
$$

and pairwise orthogonal minimal tripotents $e_{j}$ in $E$. For every $j \leq r$ the number $\sigma_{j}(a):=\sigma_{j}$ is called the $j^{\text {th }}$ singular value of $a$. For convenience we put $\sigma_{j}(a)=0$ for all $j>r$. Then clearly every element $z$ in the orbit $M=K(a)$ has finite rank $r$ and $\sigma_{j}(z)=\sigma_{j}(a)$ for all $j$. The minimal tripotents in $E$ are precisely the unit vectors of rank 1 .

Denote by $\mathcal{F}$ the subspace of all finite rank elements in $E$, which is closed in $E$ if and only if it has finite dimension. $\mathcal{F}$ is an ideal in $E$ and there exist pairwise different minimal ideals $\mathcal{I}_{1}, \ldots, \mathcal{I}_{p}$ of $\mathcal{F}$ such that $a \in \mathcal{I}_{1} \oplus \ldots \oplus \mathcal{I}_{p}$, i.e. $a=a_{1}+\ldots+a_{p}$ with $0 \neq a_{k} \in \mathcal{I}_{k}$. But then the orbit $M=K(a)$ also is a direct product $M=M_{1} \times \ldots \times M_{p}$ of orbits $M_{k}:=K\left(a_{k}\right)$ of positive dimensions. As a consequence, it is not a real restriction to assume in the following that $\mathcal{F}$ itself is a minimal ideal in $E$. But then the closure $\mathcal{K}$ of $\mathcal{F}$ in $E$ is an irreducible $\mathrm{JB}^{*}$-triple in which the closed linear span of all minimal tripotents is the whole space. $\mathrm{JB}^{*}$-triples of this type are called elementary Cartan factors. In generalization of (6.1) $z \in \mathcal{K}$ has a representation

$$
z=\sum_{j=1}^{\infty} \sigma_{j} c_{j}
$$

with pairwise orthogonal tripotents $c_{j}$ in $E$ and uniquely determined real coefficients

$$
\sigma_{1} \geq \sigma_{2} \geq \ldots \geq \sigma_{j} \geq \ldots \text { satisfying } \lim _{j \rightarrow \infty} \sigma_{j}=0
$$

such that $c_{j}$ is minimal if $\sigma_{j} \neq 0$ and $c_{j}=0$ otherwise. Again we put $\sigma_{j}(z):=\sigma_{j}$ for all $j$.

There exists a unique hermitian form $(z \mid w)$ on $\mathcal{F}$ with

$$
(z \mid z)=\sum_{k=1}^{\infty} \sigma_{k}(z)^{2}
$$

for all $z \in \mathcal{F}$. The corresponding norm $\|z\|_{2}=\sqrt{(z \mid z)}$ satisfies $\|z\|_{2} \geq\|z\|$ on $\mathcal{F}$. Therefore the completion $\mathcal{H} \mathcal{S}$ of $\mathcal{F}$ with respect to \|\|$_{2}$ is a complex Hilbert space that can be identified with the linear subspace

$$
\mathcal{H S}=\left\{z \in \mathcal{K}: \sum_{k=1}^{\infty} \sigma_{k}(z)^{2}<\infty\right\} .
$$


Actually, $\mathcal{H S}$ is a Hilbert $\mathrm{J}^{*}$-triple in the triple product induced from $\mathcal{K}$ and $\operatorname{Aut}(\mathcal{H S})=\operatorname{Aut}(\mathcal{K})$ for the corresponding automorphism groups.

For the study of finite rank orbits in $E$ it will be enough to study the case where $E$ is an elementary Cartan factor. Since the finite dimensional case is already taken care of in [13] we may even restrict to the infinite dimensional case. The elementary Cartan factors are completely classified: besides two exceptional types $\mathbf{V}$ and $\mathbf{V I}$ in dimensions 16 and 27 there are precisely the following four types I - IV. For their description let $1 \leq m \leq n$ be arbitrary cardinal numbers. Let furthermore $L$ and $H$ be complex Hilbert spaces of dimensions $m$ and $n$ respectively. On $H$ we fix a conjugation $x \mapsto \bar{x}$ (i.e. a conjugate linear involutory isometry). The unitary group of all surjective linear isometries of $H$ is denoted by $\mathrm{U}(H)$.

Type $\mathbf{I}_{m, n}: \quad E=\mathcal{K}(L, H)$ is the space of all compact linear operators $L \rightarrow H$ with triple product $\{a b c\}=\left(a b^{*} c+c b^{*} a\right) / 2$ where $b^{*} \in \mathcal{K}(H, L)$ is the adjoint of $b$. For every $z \in E$ the triple rank coincides with the operator rank of $z$, that is, with the dimension of its range. $\mathcal{H S}$ is the subspace of all Hilbert-Schmid operators in $E$. The group $K=\operatorname{Aut}(E)^{0}$ consists of all transformations $z \mapsto u z v$ with $v \in \mathrm{U}(L)$ and $u \in \mathrm{U}(H)$, the group $\Gamma=\operatorname{Str}(E)^{0}$ consists of all transformations $z \mapsto x z y$ with $y \in \mathrm{GL}(L)$ and $x \in \mathrm{GL}(H)$.

Type $\mathbf{I I}_{n}: E=\left\{z \in \mathcal{K}(H): z^{\prime}=-z\right\}$, where the transpose $z^{\prime}: H \rightarrow H$ is defined by $z^{\prime}(x)=\overline{z^{*}(\bar{x})}$ for every $x \in H$. For every $z \in E$ the triple rank of $z$ is half the operator rank of $z$. The group $K=\operatorname{Aut}(E)^{0}$ consists of all transformations $z \mapsto u z u^{\prime}$ with $u \in \mathrm{U}(H)$.

Type $\mathbf{I I I}_{n}: E=\left\{z \in \mathcal{K}(H): z^{\prime}=z\right\}$. Triple rank and operator rank coincide. Again, the group $K=\operatorname{Aut}(E)^{0}$ consists of all transformations $z \mapsto$ $u z u^{\prime}$ with $u \in \mathrm{U}(H)$.

Type $\mathbf{I} \mathbf{V}_{n}$ : for $\operatorname{dim}(H) \geq 3, E$ is the Hilbert space $H$ endowed with the equivalent norm \|\| defined by

$$
\|z\|^{2}=(z \mid z)+\sqrt{(z \mid z)^{2}-|\langle z \mid z\rangle|^{2}}
$$

and triple product

$$
\{x y z\}:=(x \mid y) z+(z \mid y) x-\langle x \mid z\rangle \bar{y},
$$

where $\langle z \mid w\rangle:=(z \mid \bar{w})$ defines a symmetric bilinear form $\langle\mid\rangle$ on $H$. Every $z \in E$ has rank $\leq 2$, and $z$ has rank $\leq 1$ if and only of $\langle z \mid z\rangle=0$. Clearly, $X:=\{z \in H: \bar{z}=z\}$ is a real Hilbert space and the orthogonal group $\mathrm{O}(X)$ of all surjective $\mathbb{R}$-linear isometries of $X$ can be considered in a canonical way as a subgroup of $\mathrm{U}(H)$. The group $K=\operatorname{Aut}(E)^{0}$ consists of all transformations $e^{i t} \lambda$ with $t \in \mathbb{R}$ and $\lambda \in \mathrm{O}(X)$. The Hilbert space $\mathcal{H S}$ is nothing but $H$ with its original norm.

For the following let us assume that $E$ is an elementary Cartan factor. The following statements are well known in the finite dimensional case and can easily be seen by checking every infinite dimensional case separately. For every integer $r \geq 1$ let us call every length-r-sequence $e_{1}, \ldots, e_{r}$ an $r$-frame in $E$ if the members are pairwise orthogonal minimal tripotents in $E$. 
Lemma 6.2. Let $e_{1}, \ldots, e_{r}$ and $c_{1}, \ldots, c_{r}$ be two $r$-frames in $E$. Then there exists a transformation $g \in K$ with

(i) $g\left(e_{k}\right)=c_{k}$ for $k=1, \ldots, r$,

(ii) $g(x)=x$ for all $x \in E$ with $e_{k} \perp x \perp c_{k}$ for all $k$.

In particular, the group $K$ acts transitively on the space of all $r$-frames in $E$.

Lemma 6.3. For every integer $r \geq 0$ the set $\mathcal{F}_{r}$ of all elements of rank $\leq r$ in $E$ is closed in $E$ as well as in the Hilbert space $\mathcal{H S}$. Actually, there exists a set $\mathcal{P}$ of holomorphic homogeneous polynomials of degree $r+1$ on $E$ with

$$
\mathcal{F}_{r}=\{z \in E: f(z)=0 \text { for all } f \in \mathcal{P}\} .
$$

LemMa 6.4. For every $a \in E$ of finite rank the orbit $M=K(a)$ is given by

$$
M=\left\{z \in E: \sigma_{k}(z)=\sigma_{k}(a) \text { for all } k\right\}
$$

and is also a closed direct real-analytic submanifold of the complex Hilbert space $\mathcal{H S}$. In particular, $\mathcal{H S}$ induces on $M$ a $K$-invariant Riemannian metric.

Lemma 6.2 is not true in general for infinite sequences $\left(e_{k}\right)_{k \geq 1},\left(c_{k}\right)_{k \geq 1}$ of pairwise orthogonal minimal tripotents. Counter examples occur in case $n=\aleph_{0}$ and $E$ of type $\mathbf{I}_{n, n}, \mathbf{I I}_{n}$ or $\mathbf{I I I}_{n}$ : choose $\left(e_{k}\right)$ in such a way that $\{z \in E: z \perp$ $e_{k}$ for all $\left.k\right\}=\{0\}$. Then there does not exist a transformation $g \in K$ with $g\left(e_{k}\right)=e_{k+1}$ for all $k$. Furthermore, for every strictly decreasing sequence $\left(\sigma_{k}\right)$ in $\mathbb{R}$ with $\lim _{k \rightarrow \infty} \sigma_{k}=0$ the elements $a:=\sum \sigma_{k} e_{k}$ and $b:=\sum \sigma_{k} e_{k+1}$ in $E$ have the same singular values but satisfy $b \notin M=K(a)$, that is, Lemma 6.4 also is not true if $a$ is not of finite rank.

\section{7. - Holomorphic structure of finite rank orbits}

Suppose $a$ is an algebraic element in the JB*-triple $E$. Then for $K=$ $\operatorname{Aut}(E)^{0}$ and $\Gamma=\operatorname{Str}(E)^{0}$ the corresponding orbits $M=K(a)$ and $X=\Gamma(a)$ are direct submanifolds of $E$, more precisely, $X$ is a complex manifold and $M$ is a closed real analytic submanifold of $X$ with $T_{x} X=T_{x} M+i T_{x} M$ for all $x \in M$.

$M$ has a natural Cauchy-Riemann structure that is given by the distribution of the so called holomorphic tangent spaces $H_{x} M:=T_{x} M \cap i T_{x} M, x \in M$, together with the complex structure on every $H_{x} M$. As in the finite dimensional setting, for every complex Banach space $F$, a smooth function $f: M \rightarrow F$ is called $C R$, if for every $x \in M$ the differential $d f_{x}: T_{x} M \rightarrow F$ is complex linear on the complex subspace $H_{x} M \subset T_{x} M$. A continuous function $M \rightarrow F$ is called $C R$, if it is locally the uniform limit of a sequence of smooth CR-functions. As an example, every holomorphic function defined in an open neighbourhood of $M \subset E$ gives by restriction a real-analytic CR-function on $M$. 
Natural questions about the CR-submanifold $M \subset E$ are for instance: What is the (linear, polynomial, holomorphic) convex hull of $M$ in $E$ ? To which subsets of $E$ can every continuous $C R$-function on $M$ be holomorphically extended, and in what sense? Complete answers in the finite dimensional case with $F=\mathbb{C}$ have been given in [13]. In the following we want to extend some of these results to the infinite dimensional setting, at least for elements of finite rank.

We assume for the rest of the section that $E$ is an elementary Cartan factor and $\mathcal{F}:=\mathcal{F}(E)$ is the ideal of all finite rank elements in $E$. We start with the following statement that allows reduction arguments to the finite dimensional case.

Lemma 7.1. To every linear subspace $V \subset \mathcal{F}$ of finite dimension there exists an irreducible $J B^{*}$-subtriple $U \subset \mathcal{F}$ of finite dimension with $V \subset U$.

Proof. Type I: here $E=\mathcal{K}(L, H)$ for complex Hilbert spaces $L, H$. There exist linear subspaces $L_{1} \subset L$ and $H_{1} \subset H$ of finite dimensions with $V(L) \subset H_{1}$ and $V^{*}(H) \subset L_{1}$. Then

$$
U:=\left\{z \in E: z(L) \subset H_{1}, z^{*}(H) \subset L_{1}\right\}
$$

is a $\mathrm{JB}^{*}$-subtriple of $\mathcal{F}$ isomorphic to the irreducible $\mathrm{JB}^{*}$-triple $\mathcal{L}\left(L_{1}, H_{1}\right)$ of finite dimension.

Types II, III: for $\varepsilon= \pm 1$ we have $E=\left\{z \in \mathcal{K}(H): z^{\prime}=\varepsilon z\right\}$, where $H$ is a complex Hilbert space with conjugation $x \mapsto \bar{x}$. We may assume that $H$ has infinite dimension. Then there exists a conjugation invariant linear subspace $H_{1} \subset H$ of finite dimension $\geq 5$ with $V(H) \subset H_{1}$. Then put

$$
U:=\left\{z \in E: z(H) \subset H_{1}\right\} .
$$

Type IV: without loss of generality we may assume that $V \subset E$ is conjugation invariant and has dimension $\geq 3$. But then $V$ is a subtriple isomorphic to $\mathbf{I V}{ }_{d}$ for $d:=\operatorname{dim} V$.

Now fix an element $a \in E$ of finite rank, say $r \in \mathbb{N}$. As before let $X:=\Gamma(a)$ and $M:=K(a)$. Then $X \subset \mathcal{F}$ consists of all elements of rank $r$ in $E$, and the closure of $X$ in $E$ is the algebraic subset $\mathcal{F}_{r}$ of all elements of rank $\leq r$ in $E$. According to Section 6 let $\sigma_{j}: E \rightarrow \mathbb{R}$ be the $j^{\text {th }}$ singular value map for every integer $j \geq 1$. Then

$$
M=\left\{z \in E: \sigma_{j}(z)=\sigma_{j}(a) \text { for all } j\right\},
$$

since $a$ is algebraic. For every $j \geq 1$ define on $E$ real valued functions

$$
\begin{aligned}
& \alpha_{j}:=\sigma_{1}+\sigma_{2}+\ldots+\sigma_{j} \text { (additive variant) } \\
& \mu_{j}:=\sigma_{1} \cdot \sigma_{2} \cdot \ldots \sigma_{j} \quad \text { (multiplicative variant). }
\end{aligned}
$$

Then [13, Proposition 11.1] together with Lemma 7.1 immediately gives 
Proposition 7.2. The closed convex hull of the orbit $M$ is

$$
\operatorname{cch}(M)=\left\{z \in E: \alpha_{j}(z) \leq \alpha_{j}(\text { a) for all } j\}\right.
$$

The convex hull of $M$ is the intersection $\operatorname{cch}(M) \cap \mathcal{F}$.

In accordance with [13] we put

$$
\begin{aligned}
& \mathcal{Z}:=\mathcal{Z}(a):=\left\{z \in E: \mu_{j}(z) \leq \mu_{j}(a) \text { for all } j\right\} \\
& \mathcal{D}:=\mathcal{D}(a):=\left\{z \in \mathcal{Z}: \mu_{j}(z)<\mu_{j}(a) \text { for all } j \leq r\right\}
\end{aligned}
$$

Clearly, $\mathcal{Z}$ is a closed balanced subset of $\mathcal{F}_{r}$ and $\mathcal{D}$ is the interior of $\mathcal{Z}$ with respect to the space $\mathcal{F}_{r}$. Furthermore, $\mathcal{D} \cap X$ is a domain in the complex manifold $X=\mathcal{F}_{r} \backslash \mathcal{F}_{r-1}$ and also is dense in $\mathcal{Z}$.

The set $\mathcal{Z}$ has a rich structure with respect to its holomorphic arc components. Let us denote by $Q$ the set of all $z \in \mathcal{Z}$ with the property: for every integer $j \geq 1$ with $\mu_{j}(z)<\mu_{j}(a)$ the identity $\sigma_{j}(z)=\sigma_{j+1}(z)$ holds. Then it is easy to see that every holomorphic arc component of $\mathcal{Z}$ intersects $Q$ in at least one point, and we conjecture that this intersection point always is unique. At least, we give a proof for the following partial result.

Proposition 7.3. For every $c \in M$ the one-point set $\{c\}$ is a holomorphic arc component of $\mathcal{Z}$. In particular, every holomorphic map $\varphi: \Delta \rightarrow E$ with $\varphi(\Delta) \subset M$ is constant.

Proof. Suppose $\varphi: \Delta \rightarrow E$ is a holomorphic map with $\varphi(\Delta) \subset \mathcal{Z}$ and $\varphi(0)=c$. Every $\alpha_{j}$ is a $K$-invariant equivalent norm on $E$ with $\alpha_{j}(\mathcal{Z}) \leq \alpha_{j}(a)$. Therefore, by [7, p. 68],

$$
\alpha_{j}(c+\zeta(\varphi(z)-c))=\alpha_{j}(c)
$$

and hence

$$
\sigma_{j}(c+\zeta(\varphi(z)-c))=\sigma_{j}(c)=\sigma_{j}(a)
$$

for all $j \geq 1$ and all $z \in \Delta, \zeta \in \mathbb{C}$ with $2|\zeta z| \leq 1-|z|$. This implies $c+\zeta(\varphi(z)-c) \in M$ for all such $\zeta, z$. Now $M$ is contained in the sphere with radius $\|a\|_{2}$ of the complex Hilbert space $\mathcal{H S}$. Since for every $z \in \Delta$ the affine mapping $\zeta \mapsto c+\zeta(\varphi(z)-c)$ takes a neighbourhood of $0 \in \mathbb{C}$ to $M$ we get $\varphi(z)=c$.

The following result in the special case of symmetric $n \times n$-matrices in finite dimensions, that is the type III $_{n}$ with $n<\infty$ in our notation, is already contained in [15]. The general finite dimensional case is contained in [13, Theorem 11.7] and [12, Proposition 3.3], compare also [6].

Proposition 7.4. The polynomial convex hull $\operatorname{pch}(M)$ of the orbit $M$ is the set $\mathcal{Z}$. 
Proof. We may assume that $E$ is of infinite dimension. Since $\mathcal{F}_{r}$ is given by polynomial equations $\operatorname{pch}(M) \subset \mathcal{F}_{r}$ holds. In a first step assume that $z \in \operatorname{pch}(M)$ is an arbitrary element. By Lemma 7.1 there exists an irreducible subtriple $U \subset \mathcal{F}$ of finite dimension with $a, z \in U$. The singular values with respect to $U$ and $E$ coincide for elements of $U$. Therefore the intersection $M \cap U$ is the orbit of $a$ under the group $\operatorname{Aut}^{0}(U)$. Every holomorphic polynomial function $U \rightarrow \mathbb{C}$ can be extended to a holomorphic polynomial function $E \rightarrow \mathbb{C}$ since $U$ is a direct linear subspace of $E$ by finite dimensionality. Therefore [13, Theorem 11.7] implies $\mu_{j}(z) \leq \mu_{j}(a)$ for all $j$, i.e. $z \in \mathcal{Z}$.

Now assume that $x \in \mathcal{F}_{r}$ is not contained in $\operatorname{pch}(M)$. Then there exist $y \in$ $M$ and a holomorphic polynomial $f: E \rightarrow \mathbb{C}$ with $|f(x)|>|f(y)|$. By Lemma 7.1 there exists an irreducible subtriple $U \subset \mathcal{F}$ of finite dimension with $x, y \in U$ and hence $x \notin \operatorname{pch}(M \cap U)$. Again [13, Theorem 11.7] implies $\mu_{k}(x)>\mu_{k}(y)=\mu_{k}(a)$ for some $k \geq 1$, i.e. $x \notin \mathcal{Z}$.

The element $a \in E$ is called invertible if the operator $Q_{a}$ on $E$ is invertible. In case $E$ is one of the types I - III, invertibility in this sense is the same as the usual one in the operator sense. In case $E$ is of type IV, invertibility of $a$ just means that $a$ has rank 2 , or equivalently, that $\langle z \mid z\rangle \neq 0$. We can now formulate the main result of this paper.

THEOREM 7.5. Let $E$ be an elementary Cartan factor and let $a \in E$ be a noninvertible element of finite rank. Let furthermore $f$ be a continuous $C R$-function on the orbit $M=K(a)$ with values in the complex Banach space $F$. Then $f$ has a unique $F$-valued pull-back holomorphic extension to the set $\mathcal{Z}=\mathcal{Z}(a)$, and this extension takes values in the holomorphic convex hull of $f(M)$ in $F$. In particular, this extension is holomorphic on the dense subset $\mathcal{D} \cap X$ of $\mathcal{Z}$, which is open in the complex manifold $X=\Gamma(a)$. instead.

We postpone the proof of 7.5 to the next section and give some applications

COROLlaRY 7.6. $\mathrm{hch}(M)=Z(a)$ for every noninvertible $a \in E$ of finite rank and $M=K(a)$.

For a fixed integer $r \geq 1$ let $E=\mathcal{L}(L, H)=\mathcal{K}(L, H)$ where $L$ is a complex Hilbert space of finite dimension $r$ and $H$ is a complex Hilbert space of infinite dimension. Then $E=\mathcal{F}_{r}$ and every element $a \in E$ is noninvertible. Let us assume for the rest of the section that $a$ has rank $r$. Then $\mathcal{D} \subset E$ is a bounded balanced domain in $E$ with Shilov boundary $M=K(a)$ and by Theorem 7.5 every continuous CR-function $f: M \rightarrow F, F$ an arbitrary complex Banach space, has a continuous extension to $\mathcal{Z}=\overline{\mathcal{D}}$ that is holomorphic on $\mathcal{D}$. On the other hand, every continuous function on $\mathcal{Z}$ that is holomorphic on $\mathcal{D}$ gives by restriction a CR-function on $M$.

Denote by $\operatorname{Aut}_{\mathrm{CR}}(M)$ the group of all homeomorphisms $g$ of $M$ such that $g$ and $g^{-1}$ are CR. Every such $g$ extends to $\mathcal{Z}$ and gives a biholomorphic automorphism of the domain $\mathcal{D}$. On the other hand, every $g \in \operatorname{Aut}(\mathcal{D})$ (the group of all biholomorphic automorphisms of $\mathcal{D}$ ) extends holomorphically onto a neighbourhood of $\mathcal{Z}$, that is, we have: 
COROLlaRY 7.7. The groups $\operatorname{Aut}_{\mathrm{CR}}(M)$ and $\operatorname{Aut}(\mathcal{D})$ are canonically isomorphic.

\section{8. - Proof of the main result}

We start with the following simple discussion: for fixed integer $r \geq 1$ let $G$ be the group of all linear transformations

$$
\left(z_{1}, z_{2}, \ldots, z_{r}\right) \mapsto\left(t_{1} z_{\pi(1)}, t_{2} z_{\pi(2)}, \ldots, t_{r} z_{\pi(r)}\right)
$$

of $\mathbb{C}^{r}$, where $t_{1}, \ldots, t_{r}$ run over all unimodular complex numbers and $\pi \in \mathfrak{S}_{r}$ is an arbitrary permutation. Fix a point $a=\left(a_{1}, \ldots, a_{r}\right) \in \mathbb{R}^{r}$ with $a_{1} \geq a_{2} \geq$ $\ldots \geq a_{r}>0$ and let $N:=G(a)$ be the corresponding orbit of $a$, which is a (not necessarily connected) real-analytic compact submanifold of $\mathbb{C}^{r}$. Then the polynomial convex hull $P:=\operatorname{pch}(N)$ of $N$ is the set of all $g(x)$ with $g \in G$ and $x \in \mathbb{R}^{r}$ satisfying

$$
x_{1} \geq \ldots \geq x_{r} \geq 0 \text { as well as } \Pi_{j=1}^{k} x_{j} \leq \Pi_{j=1}^{k} a_{j} \text { for all } k \leq r .
$$

Denote by $A$ the complex Banach algebra of all continuous functions on $P$ whose restriction to the interior $\stackrel{\circ}{P}$ of $P$ is holomorphic. The evaluation mapping $A \times P \rightarrow \mathbb{C}$ given by $(f, x) \mapsto f(x)$ is continuous. On the other hand, the restriction mapping $A \rightarrow \mathcal{C}(N)$ is a linear isometry. In particular, as a consequence of Hahn-Banach, to every $y \in P$ there exists a regular Borel measure $\mu_{y}$ on $N$ with $f(y)=\int_{N} f d \mu_{y}$ for all $f \in A$. Unfortunately, $\mu_{y}$ is not uniquely determined by $y$ in general.

Now we resume the notation from the last section: $E$ is an elementary Cartan factor and $a \in E$ is a noninvertible element of finite rank $r \geq 1$. Then $a$ has a representation

$$
a=a_{1} e_{1}+\ldots+a_{r} e_{r}
$$

with real coefficients $a_{j}=\sigma_{j}(a)$ and a suitable $r$-frame $e_{1}, \ldots, e_{r}$ in $E$. Identify $\mathbb{C}^{r}$ with the subtriple $\left\{z_{1} e_{1}+\ldots+z_{r} e_{r}: z_{k} \in \mathbb{C}\right\}$ of $E$ in the obvious way. For $N$ and $P$ as above we have $N=M \cap \mathbb{C}^{r}$ and $P=\mathcal{Z} \cap \mathbb{C}^{r}$. If we identify (by restriction) the Banach algebra $A$ with a subalgebra of $\mathcal{C}(N)$ we claim in a first step:

LEMMA 8.1. For every continuous $C R$-function $f: M \rightarrow \mathbb{C}$ the restriction $\left.f\right|_{N}$ is contained in the algebra $A$.

Proof. Choose an irreducible subtriple $U \subset \mathcal{F}$ of finite dimension with $\mathbb{C}^{r} \subset U$ such that $a$ also is noninvertible in $U$. Then the restriction $h:=\left.f\right|_{M \cap U}$ of $f$ to the Aut $(U)^{0}$-orbit $M \cap U$ is CR. By [13, Proposition 12.3] $h$ has a continuous extension to $\mathcal{Z} \cap U$ that is holomorphic on $\mathcal{D} \cap U \backslash\{0\}$. Because 
of $\mathcal{Z} \cap \mathbb{C}^{r}=P$ this implies that $\left.f\right|_{N}$ has a continuous extension to $P$ that is holomorphic on $\stackrel{\circ}{P} \backslash\{0\}$. But then by Riemann's extension theorem continuity already implies holomorphy on all of $\stackrel{\circ}{P}$.

For the next statement let us fix the continuous CR-function $f: M \rightarrow \mathbb{C}$. For every $g \in K$ the function $f_{g}:=\left.(f \circ g)\right|_{N}$ is in the algebra $A$ by Lemma 8.1, and $f_{g}(x) \in \mathbb{C}$ is jointly continuous in the parameters $g \in K, x \in P$.

Lemma 8.2. There exists a unique continuous function $\tilde{f}: \mathcal{Z} \rightarrow \mathbb{C}$ with

$$
\tilde{f}(g(x))=f_{g}(x) \text { for all } g \in K, x \in P .
$$

ProOF. Because of $\mathcal{Z}=K(P)$ we only have to show that (8.3) produces a well defined function $\tilde{f}$ on $\mathcal{Z}$, that is, we have to show

$$
f_{g}(x)=f_{h}(y) \text { for all } g, h \in K \text { and } x, y \in P \text { with } g(x)=h(y) .
$$

Without loss of generality we may assume that $h \in K$ is the identity. Choose an irreducible $\mathrm{JB}^{*}$-subtriple $U \subset \mathcal{F}$ of finite dimension that contains the subtriples $\mathbb{C}^{r}$ and $g\left(\mathbb{C}^{r}\right)$. By 6.2 there exists an automorphism $\varphi \in \operatorname{Aut}^{0}(U)$ with $\varphi\left(e_{j}\right)=$ $g\left(e_{j}\right)$ for $1 \leq j \leq r$. This implies $f_{g}=f_{\varphi}$. By [13, Proposition 12.3] the restriction of $f$ to $M \cap U$ allows a unique continuous extension $\hat{f}$ to $\mathcal{Z} \cap U$ which is holomorphic on $\mathcal{D} \cap U \backslash\{0\}$. This implies $f_{h}(y)=\hat{f}(y)=f_{\varphi}(x)=f_{g}(x)$.

Clearly, the continuous function $\tilde{f}$ in 8.2 coincides with $f$ on $M$.

LEMMA 8.4. $\tilde{f}$ is pull-back holomorphic on $\mathcal{Z}$.

Proof. Consider an arbitrary element $c \in \mathcal{D} \cap X$, where $X=\Gamma(a)$ is the complex manifold of all rank-r-elements in $E$. The tangent space $T_{c} X=$ $E_{1}(c) \oplus E_{1 / 2}(c)$ is contained in $\mathcal{F}$. We claim that the complex directional derivative $\lim _{t \rightarrow 0}(\tilde{f}(c+t v)-\tilde{f}(c)) / t$ exists for every $v \in T_{c} X$, where $t$ in the limit runs in $\mathbb{C}^{*}$. Indeed, choose an irreducible subtriple of $\mathcal{F}$ of finite dimension containing $c, v$ - then the restriction of $\tilde{f}$ to the complex manifold $\mathcal{D} \cap X \cap U$ is holomorphic. As a consequence, the restriction of $\tilde{f}$ to $\mathcal{D} \cap X$ is Gateaux-holomorphic and hence holomorphic because of continuity, compare for instance [7, p. 43].

Now let $Z$ be an arbitrary connected complex Banach manifold and let $\varphi: Z \rightarrow E$ be a holomorphic mapping with $\varphi(Z) \subset \mathcal{Z}$. We have to show that the function $h:=\tilde{f} \circ \varphi$ is holomorphic on $Z$.

CASE 1. $\varphi(Z)$ contained in $\mathcal{D}$ but not in $\mathcal{F}_{r-1}$. Since $\mathcal{F}_{r-1}$ is defined by a family of holomorphic equations in $E$ there exists a non-constant holomorphic function $g$ on $Z$ such that $h$ is holomorphic on $\{z \in Z: g(z) \neq 0\}$. Since $h$ is continuous we derive that $h$ is holomorphic on $Z$.

CASE 2. $\varphi(Z)$ contained in $\mathcal{D} \cap \mathcal{F}_{r-1}$. Choose $k \leq r-1$ maximal with $\varphi(Z) \subset \mathcal{F}_{k}$. Since holomorphy of $h$ is a local property, we may assume 
without loss of generality that there exists a tripotent $v$ of rank $r-k$ in $E$ that is orthogonal to all elements of $\varphi(Z)$. By the same reasoning we may assume furthermore that for suitable $\varepsilon>0$ all functions $h_{t}(z):=h(z)+t v, 0<t<\varepsilon$, on $Z$ have range in $\mathcal{D}$. Then by Case 1 every function $h_{t}$ is holomorphic on $Z$, implying that also $h=\lim _{t \rightarrow 0} h_{t}$ is holomorphic.

CASE 3. $\varphi(Z)$ not contained in $\mathcal{D}$. For every $0<t<1$ the function $h_{t}:=t h$ on $Z$ has image in $\mathcal{D}$ and hence is holomorphic by Case 1 . Then also $h=\lim _{t \rightarrow 1} h_{t}$ is holomorphic on $Z$.

We are now ready for the

Proof of THEOREM 7.5. By the considerations above the scalar function $\lambda \circ f$ can be considered as a pull-back holomorphic function on $\mathcal{Z}$ for every $\lambda \in F^{*}=\mathcal{L}(F, \mathbb{C})$. Consider $\mathbb{C}^{r} \subset \mathcal{F}, N:=M \cap \mathbb{C}^{r}$ and $P:=\mathcal{Z} \cap \mathbb{C}^{r}$ as above. Then $N$ is compact and for every $y \in \mathcal{Z}$ and every $\lambda \in F^{*}$

$$
|(\lambda \circ f)(y)| \leq\|\lambda\| \cdot \sup \|f(g(N))\|<\infty
$$

holds, where $g \in K$ is an arbitrary transformation with $y=g(x)$ for some $x \in P$. In particular,

$$
\lambda \mapsto(\lambda \circ f)(y)
$$

defines an element $\hat{f}(y) \in F^{* *}$. We obtain this way a continuous map $\hat{f}: \mathcal{Z} \rightarrow$ $F^{* *}$ that coincides on $M$ with $f$. We claim that every $\tau:=\hat{f}(y)$ actually is in $F$. Indeed, we may assume $y \in P$ and choose a regular Borel measure $\mu_{y}$ on $N$ with $h(y)=\int_{N} h d \mu_{y}$ for all $h \in A$. Then the integral $q:=\int_{N} f d \mu_{y} \in F$ is well defined, compare [4, p. 80], and satisfies

$$
\lambda(q)=\int_{N}(\lambda \circ f) d \mu_{y}=(\lambda \circ f)(y)=\tau(\lambda)
$$

for all $\lambda \in F^{*}$, that is, $\tau=q \in F$ and hence also $\lambda \circ \hat{f}=\lambda \circ f$ for all $\lambda \in F^{*}$.

Let $Z$ be an arbitrary complex Banach manifold and let $\varphi: Z \rightarrow E$ be a holomorphic mapping with $\varphi(Z) \subset \mathcal{Z}$. Then $h:=\hat{f} \circ \varphi$ is continuous and $\lambda \circ h$ is holomorphic on $Z$ for every $\lambda \in F^{*}$. But this is enough for $h$ being holomorphic itself, compare for instance [7, p. 43]. This implies that $\hat{f}$ is pull-back holomorphic on $\mathcal{Z}$.

\section{REFERENCES}

[1] M. S. Baouend - P. Ebenfelt - L. P. Rothschild, "Real Submanifolds in Complex Spaces and Their Mappings”, Princeton Math. Series 47, Princeton Univ. Press, 1998.

[2] M. S. BAouendi - F. TReves, A property of the functions and distributions annihilated by a locally integrable system of complex vector fields, Ann. of Math. (2) 113 (1981), 387-421.

[3] A. Boggess, "CR Manifolds and the Tangential Cauchy-Riemann Complex", Studies in Advanced Mathematics, CRC Press. Boca Raton, Ann Arbor, Boston, London 1991. 
[4] N. BoubaKi, "Integration", Hermann, Paris 1965.

[5] S. DineEn, "Complex Analysis on Infinite Dimensional Spaces", Berlin-Heidelberg-New York, Springer, 1999.

[6] J. Faraut - L. Bouattour, Enveloppes polynômiales d'ensembles compacts invariants, Math. Nachr. 266 (2004), 20-26.

[7] T. Franzoni - E. Vesentini, "Holomorphic Maps and Invariant Distances", North Holland, Amsterdam, 1980.

[8] L. A. HARRIS - W. KAUP, Linear algebraic groups in infinite dimensions, Illinois J. Math. 21 (1977), 666-674.

[9] W. Kaup, Algebraic Characterization of Symmetric Complex Banach Manifolds, Math. Ann. 228 (1977), 39-64.

[10] W. Kaup, A Riemann Mapping Theorem for Bounded Symmetric Domains in Complex Banach Spaces, Math. Z. 183 (1983), 503-529.

[11] W. Kaup, On spectral and singular values in JB*-triples, Proc. Roy. Irish. Acad. 96A (1996), 95-103.

[12] W. KAUP, Bounded symmetric domains and polynomial convexity, Manuscripta Math. 114 (2004), 391-398.

[13] W. KAup - D. ZAitsev, On the CR-structure of compact group orbits associated with bounded symmetric domains, Invent. Math. 153 (2003), 45-104.

[14] O. Loos, "Jordan pairs", Springer Lecture Notes 460, 1975.

[15] C. SACRÉ, Enveloppes polynomiales de compacts, Bull. Sci. Math. 116 (1992), 129-144.

[16] J. SAUteR, "Randstrukturen beschränkter symmetrischer Gebiete", Dissertation, Tübingen, 1995.

Mathematisches Institut Universität Tübingen Auf der Morgenstelle 10 D-72076 Tübingen, Germany kaup@uni-tuebingen.de 